\title{
Multi-Hop Video Routing Methods: State of the Art
}

\author{
Vaibhav Mishra \\ M.Tech. Scholar \\ Jodhpur Institute of Engineering \\ and Technology
}

\author{
Kavita Choudhary \\ Assistant Professor (Sr.) \\ Jodhpur Institute of Engineering \\ and Technology
}

\author{
Saurabh Maheshwari \\ Assistant Professor \\ (Student Member IEEE) \\ Govt. Women Engineering \\ College Ajmer, India
}

\begin{abstract}
In this paper video transmission in the Internet scenario, where packets move from source to destination, has been studied. In this various methods and schemes are discussed to transmit the packet from sender to destination efficiently. Various methods like encryption, scrambling techniques are used to hide the content from the intruders. These algorithms are compared on various parameters like delay, PSNR, throughput to provide the smooth playback of video at the destination. These algorithms are efficient to transmit the video data securely from sender to destination. Still the problem of video copy attack exists where an intruder can copy all the coming packets and extract frames from them. If the sequence of packets is same as sequence of frames then whole video can be thus copied. In future algorithms preventing the packet copy attack on the video data in the network.
\end{abstract}

\section{General Terms}

Multi-hop routing, video quality, video encryption

\section{Keywords}

Multi hop, Multipath, dual channel

\section{INTRODUCTION}

The multi hop video routing methods are needed to reduce the malicious attacks to protect the precious content from the intruders. The efficiency of the algorithm is increased in multi hop network because when the data is sent from sender to receiver, the delay decreases and possibility of dropping the packets is also reduced. The throughput of the algorithm increases in the multi hop network without compromising the security of the video data. It has various applications like video on demand; video transmission over IP networks, video encryption, IP network multi-hop routing, secure virtual paths, and IPTV security. In the recent scenario, the data sent by the sender moves from the network, to the receiver. In network many intermediate nodes are present which passes the packets to other nodes in a fixed path so that packet can reach to the destination. Therefore there is a probability that some nodes which are present between source and destination behaves maliciously. The motive of these malicious nodes is to leak important information which is passed through it in the form of packets. The data can be in any form like text, audio, video, image. In case of video data, a problem arises for the secure transmission of the video from source to destination therefore multi hop video routing methods are essential to send the video data securely from sender to receiver. Some of the benefits of the multi hop video routing methods are simultaneous, parallel transport over multiple channels, load balancing over available resources. The path discovery on reassigning an interrupted stream by continuously offering packets to all paths is avoided and hence the paths are fully utilized. The paper is structured in various sections. In section
2, security of the IPTV is described in which different methods are explained to provide the security to the content of the IPTV. In section 3, the video encryption is described in which different methods and techniques are mentioned to provide the security to the content and reduce the overhead from encoder for encryption and decryption. In section 4, video streaming is explained in which video data is coming from the network and packets are collected at the receiver for the smooth playback of the video at the receiver side. In section 5, multi-channel concept is introduced in which different methods are described to send the video data from sender to destination efficiently. In section 6, real time constraints are explained which affect the video data in the network like delay, load balancing, reliable delivery and quality of service. Section 7 introduces the MAC layer in which routing frame work is described.

\section{SECURITY FOR IPTV}

\subsection{Content and Service Protection}

IPTV provides a good quality video as compare to the conventional television. The IPTV is not completely different from the digital TV. The main difference is the bidirectional communication process of IPTV. In the recent scenario it is a topic of research in which IPTV is analyzed on the various security issues. There are the different security issues like content security threats which contain masquerading, network security threats, terminal device security threats, subscriber security threats, terminal device security threats, and subscriber security threats. There are different security requirements which are essential to provide the security to the content of the IPTV. Initially an identification process is required so that each entity of an IPTV provides unique identifier. This will provide the end to end security to the IPTV content. In authentication, the identified element of the content is certified. In authorization, the service provider gives permission to access the content. In key distribution, keys are reached to the destination with respect to the encrypted data so that the receiver cannot wait for the decryption of the encrypted data. In content encryption, the scrambling technique is used for encrypting the content of the television. In IPTV, the encryption is done to provide the security to the content. In right expression, pre TV flags are used to indicate the access control rights for the content of the IPTV. In renewability and revocation, some of the offending elements on the television content are replaced by the contents with a more robust method. In compliance and robustness regime, the protection of the content security of a system is required which is robust for the protection of content from hackers and intruders. There are some solutions provided for the security of the IPTV content like scalable key management scheme which is used to handle many members without any degradation in the performance. Also, Watermarking technique provides security to the data by adding a watermark symbol to the digital data. Fingerprinting 
technology is used for the access control. The researchers examine that up to which extent older technologies are replaced by newer one in cost effective manner $[1,2]$.

\section{VIDEO ENCRYPTION 3.1 Scalable Frame Scrambling}

Various encryption schemes are present to encrypt the video data but some of the schemes degrade the quality of the video data. Therefore, there is a need of an encryption scheme which encrypts the data without affecting the quality of the video. In this scheme, first the sequence of the ordered frames is broken into the frame sequences and then these frames are scrambled internally with the help of keys. Each frame sequences consist of the arbitrary number of frames. This scheme is applicable only to the long duration videos which require less security. This scheme is cost effective as there is no change in the content of the frames. For videos, video frames are broken into frame sequences and then these frame sequences are internally scrambled with the help of the scrambling key. The pixels of frames are scrambled with the pixels of other frames to prevent the look up of the contents. It shows that scheme of frames distribution gives a fact that video is only useful when all the frames are in correct order [3].

\subsection{Selective Encryption}

\subsubsection{Video Bit Streams}

The video bit streams present the advancement in the existing encryption techniques as compared to the previous techniques. In this, three new selective encryption methods are introduced. In the first method, the encryption process is applied on the data of all intra macro blocks. In the second method, the encryption is applied on both the headers of the micro block and the data of every intra macro block. Third method is applied on the macro blocks to enhance the security and reduce the total encryption time. In this the DES key is used for the encryption. An alternative method for encrypting the intra coded macro blocks is introduced in which a counter is maintained which is increased by one when the encryption of the macro block is done. Still some of the macro blocks left unencrypted but these macro blocks cannot give any important information because differential encoding scheme is used for encrypting the macro blocks. The simulated results show that encryption of all the intra macro blocks and predicted macro blocks prevent the wastage of the processing time [5].

\subsubsection{Compressed Bit Streams}

The compressed bit streams format MPEG-1 requires large amount of processing. The existing methods are not suitable for encrypting these bit streams therefore a new scheme is introduced in which Intra coded macro blocks are encrypted with the data present in them. The two new methods are applied for the selective encryption. In the first method all the Intra coded Macro blocks are encrypted and in the second method the headers and data of the Intra coded macro blocks are encrypted. DES key is used in both the methods for encryption purpose. The DES key changed periodically and transmitted in the encrypted form to prevent the brute force attack from the key. The encryption of the key is done by the public key cryptosystem which uses the RSA standard. Only I-MBis encrypted to provide security to all the frames in the intra coded macro blocks [4].

\subsection{Secret Key Cryptography}

The security of the multimedia applications is very important for secure transmission of video from source to destination. There are existing algorithms like DES and IDEA for the encryption of the data. These algorithms have complex computations and the software implementation is not fast to encrypt the vast amount of data. There are many challenges present in the encryption of the video. First challenge is to encrypt the large size of the video. Second, processing the huge amount of data creates the big burden over a video codec. All these issues are removed by introducing the new scheme which encrypts the MPEG video. The encryption of the motion vectors and selected bits of DCT coefficients is done which are signed with the help of the secret key. This algorithm is efficient because it can create small overhead to the video codec therefore the compression of the MPEG video is done easily. Implementation of this algorithm is fast therefore it fulfills the requirement of the MPEG video application. The RVEA algorithm is best for both cipher text and plain text attack for the different size of the video frame. RVEA algorithm encrypts the selected bits of the video rather than encrypting the whole video. This makes it faster than other standard algorithms like IDEA and DES. The encryption and decryption of the video is fast enough so that video data cannot suffer from jitter. The video frames which are larger in size increases the delay which results in the degradation of quality of the video playback.

\subsection{Frequency Transform}

In recent scenario, the security of the multimedia application is very important. The services of multimedia like video on demand and video conferencing requires security so that malicious intruders cannot get the precious data as for video on demand service, user pays for the video. There are existing algorithms present for encryption of the video like DES but the software implementation of the algorithm is not fast that is the basic requirement for real time video application. These algorithms also take longer time for encrypting the video. Hence, a new algorithm is introduced which has secret key for encryption in which sign bits are randomly encoded by the DCT coefficients of I- pictures and the motion vectors encoded sign bits of $\mathrm{B}$ and $\mathrm{P}$ pictures. The addition of overhead in the video codec is less [18]

\subsubsection{Inter Block Frame Scrambling}

The main motive of encryption algorithm is to prevent the data from the unauthorized users. The multimedia application has high information rate and low information value therefore light weight approach is required for the encryption and decryption of the video. The experimental results show that when motion vectors of $\mathrm{B}$ and $\mathrm{P}$ frames are encrypted, the image of video is blurred but the video is comprehensible and on encrypting the I-frames of DC coefficients, the video image is obscured but still motion directions are clearly shown. The implementation of the VEA algorithm in software is fast to achieve the requirement of the real time video application and provides security to MPEG video applications efficiently [18].

\subsubsection{Intra Block Pixel Scrambling}

The development of the multimedia services like video on demand and pay per use demand security to protect data from malicious attackers. There are various techniques present like encryption, scrambling, zigzag permutation which makes the video unrecognizable for the intruders. Some service providers need the video data to be completely unrecognized for the third person. They can watch the degraded quality of 
the video so that in future they can connect with the service provider for the services. For achieving this motive, a partial scrambling algorithm is applied. The transparent scrambling technique performs the linear transformation on every pixel of RGB value. In this, the descrambler follows the decoder for decoding the scrambled images. This scheme has few advantages but still drawbacks are present for the decoder. This technique performs the linear transformation on every pixel in RGB format therefore a little overhead increases for hardware utilization. The throughput of this technique is better that the previous techniques due to less computation overhead on encrypting the intra blocks [21]. A scrambling scheme scrambles the compressed video data. This scheme is needed due to increase in the security attacks on applications like video-on-demand, video conference and video chatting system. The quality of the image is good. The secret key is present to prevent the known plain text attack [28].

\subsection{Perceptibility}

As the video data is widely used in various applications, it is very important to provide the security to video data. The security is provided by encrypting the video data. The encryption of video in pay per view and video on demand is called perceptual encryption. The aim of this encryption is that some videos which contain the degraded quality of sound can listen the sound properly. This encryption has control factor $\mathrm{P}$ which is used to control the presence of noise in the video data therefore a new encryption scheme is introduced which encrypts the code words which has fixed length in the MPEG video. This encryption scheme can works efficiently with block cipher or stream cipher [20].

\subsection{Partial Encryption}

When the video is transmitted from source to destination, the video is first encrypted. Initially in the existing algorithms the decryption of the video takes large computation time and the smooth playback of the video is hindered. When the additional burden increases in the device the frames are dropped. Therefore to reduce this problem, a new scheme is proposed which partially encrypts the data. The encryption is done by symmetric key. The level of the selection is at sample level not at sub sample level and only these samples are encrypted. The implementation of the partial encryption scheme on the device takes minimal time. This algorithm contributes in two ways. Firstly, it encrypts the audio-video by less hindering the security of that video and secondly, it provides a hierarchical model which has tunable variables and immediate results of partial encryption scheme [25].

\section{VIDEO STREAMING}

\subsection{Multi-Hop Wireless Networks}

The lot of work has been done for the transmission of the video over multi hop wireless networks in which the quality of the video is measured on the different parameters like throughput delay and packet loss rate but there is not any method present which deals with video as routing metric for transmission of video in real time. Therefore an application centric specific routing scheme is designed for video transmission. In this scheme the distortion of the video is calculated very fast and the optimization of the cross layer improves the strength of routing. This approach gives the best performance on existing networks as compared to other schemes [6].In recent scenario the opportunistic method has taken the attention as an enhanced routing metric for the transmission of the video. A conventional routing metric method selects a static path between source to destination.
Transmission can takes place anytime through the common path while in opportunistic routing scheme it chooses the nearest hop from which packet have already passed. This will increase the transmission rate per hop. The traditional routing was inefficient because they choose common path. Opportunistic scheme is better than traditional due to increase in the performance by sending the video using two hop route. Opportunistic routing increases the utilization of the network. It is a multi path routing algorithm in which each packet takes different path to reach the destination. It takes nearest hop through which packets already passed. In this, packets pass through the intermediate nodes to reach the destination and hence no one can predict the actual set of the nodes [16].

\subsection{Analytical Capacity Estimation}

There are lot of issues exists to send video data in an IEEE 802.11 based network. There is noise interference present in the network which increases the processing time of the video. The load balancing is other issue in the multi hop wireless network because for balancing the load if the longer path is taken then the delay increases and increase in the path also increases the inter and intra path interference between source and destination. A new method is introduced in which all these issues are resolved. In this approach the capacity of node is measured which is currently working and on the basis of queue models. Interference delays are also measured. The new routing path method captures the load balancing and low noise interference features which results in the good quality video streaming [14].

\section{MULTICHANNEL CONCEPT 5.1 Multichannel Multi-Hop Communication}

Today video transmission in ad hoc network is a great challenge due to the dynamic behavior and lack of infrastructure. When the video is transferred from sender to receiver through different channels, the problem of intra path interference occurs. Therefore a new scheme based on the CSMA/CA network in a single link is introduced. It reduces the interference between the paths after that multichannel scheme came in existence which stops the interference in the relay nodes from the sender to the receiver. AODV protocol is made for assigning the channel. The implementation of this scheme shows that the inter path and intra path interference depends on the transmission of data. The results show that this scheme effectively suppresses the interference among the multipath [7].

\subsection{Mobile Ad hoc Multimedia Transfer}

In recent working of video transmission from source to destination on a single path, problem of intra path interference arises which affects the performance of the network. In multipath, the video data is transmitted through the different paths. In these paths the problem of inter path interference increases which suppresses the performance of the channel. The prevention of the interference has been made by making a new scheme which works on AODV. This scheme provides better channel assignment technique than existing ones which increases the performance of multi path between source and destination. Other approach is made to send real time traffic over multipath. This routing strategy effectively manages the congestion on the network but this approach suffers from cochannel interference on the continuous transmission of packets. [8]. 


\subsection{Multiple Description Video Transmission}

Multiple description video technique is a recent technique used in wireless mesh network which has property to control error and multimedia applications in the network. This technique is different from the traditional layered technique with respect to sending the video from sender to destination. In multiple description video technique, the video are divided into streams and these streams are sent from sender to receiver and at receiver side these streams are collected to make an original video [10].In peer to peer routing, streaming of video is very difficult due to time varying nature of both the peer. This new approach has features like flexible multiple description coding and TCP friendly rate control. These features add the good throughput as compared to the other multiple description channel schemes. This algorithm has advantage that it has unicast peer to peer streaming system [29].In wireless multihop networks, the routing is done to achieve optimization of the application performance by multipath routing of the video. Multiple descriptions in a multi hop wireless network environment are made by developing a branch and bound framework. This algorithm has good performance in comparison with the other routing algorithm which follows the non-cross layer approach [30].

\subsection{Multipath Multi-Stream}

Today the video surveillance is done by using the wireless sensor networks. Video data is sent over wireless sensor network through the nodes present in the wireless sensor networks. These nodes sense the data therefore the energy constraint is attached with these networks. If the reliability of network is increased by end to end delivery it will increase the cost. Therefore by considering all these factors, a new scheme is proposed in which video data is divided into different streams. Each stream move from a different path. The energy required to send the video data from source to destination is very less [11].

\subsection{Hierarchical Routing}

Today, lot of smart applications are present in the wireless multimedia sensor networks in which nodes sense the data and takes decision according to that data. Energy constraints present in the MSN networks are much stricter than the wireless sensor networks (WSNs) because it takes more time to sense the huge amount of video data and transfer it to the destination side. The existing hierarchical routing protocols are not capable for finding path based on the energy of the network. Therefore to remove all these issues, a new hierarchical routing algorithm for efficient video transmission is used. MEVI approach has various functions like it is an opportunistic scheme in which clusters are formed with the help of sending the beacon messages.. Cross layer is used to detect the network condition on the basis of energy and quality of link for selecting the route. In MEVI cluster communications is done in two forms intra cluster communication and inter cluster communication [15].

\subsection{Multicasting}

In recent scenario wireless mesh networks and mobile ad hoc networks are widely used. In multi hop wireless network multicast traffic is used. This purpose is achieved by calculating the transport capacity. Different wireless nodes of multiple radios have the benefit of available multiple wireless channels to increase throughput. This enhanced throughput property is used widely in multicast flow in various application like video download. In a single channel multicast network, routing through the single path is still matter of research. In a multicast flow, the multicast tree is maintained in which source are acting as a root node and destinations are the leaves. Some of the multicast examples are sterner tree in which routing is optimal but it requires information in a centralize manner, therefore due to increase in the computation overhead this approach is not good. In multichannel, different channels are assigned interface with routing structure. The observation shows that smaller number of channel results in better capacity for limited number of interfaces and if number of channels increases multicast capacity decreases gradually [22].

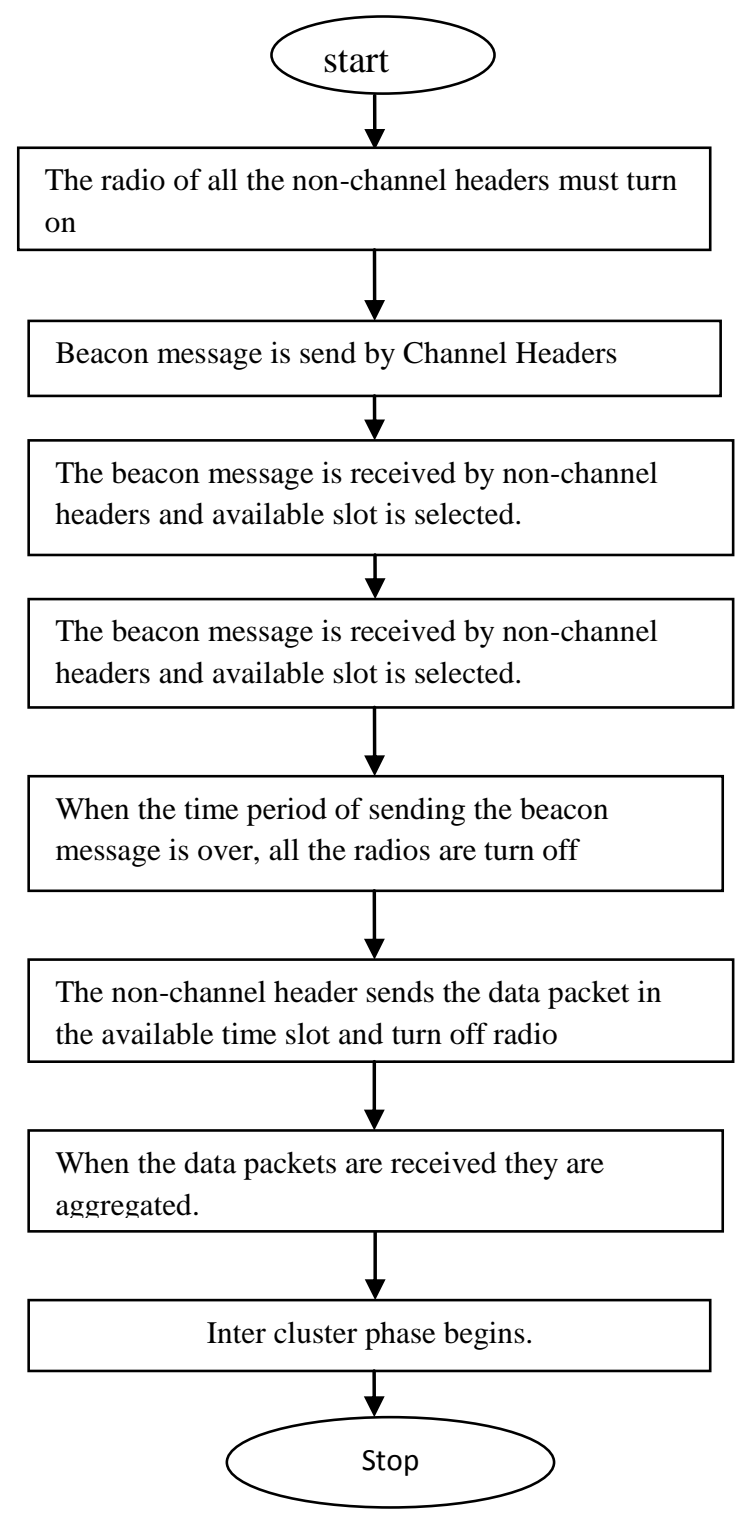

Fig 1: Flow chart of Intra cluster communication

\section{REAL TIME CONSTRAINTS 6.1 Statistical Delay Bound}

In the recent work scalable videos are transmitted from source to destination. The scalable video encoders are present which encrypts the video at different bit rates. Various problems arise in the video layering when it is sent either from a single path or from a multipath. Therefore after analyzing these problems, a problem of statistical delay bound is taken for 
further analysis which occurs when the layered video is sent from sender to receiver. The bandwidth of the different links is managed by a queuing behavior. The basic problem which arises in the multi hop transmission is resource allocation which is solved by the statistical bound [9].

\subsection{Reliable Delivery}

Today the video surveillance is done by using the wireless sensor networks. The data send from source to destination with the help of nodes present in the wireless sensor networks. The nodes sense the data therefore the energy constraint is attached with these networks. The type and the size of the video is also one of the constraints that are present while sending the video from wireless sensor network. Due to the presence of these constraints, the reliability of network decreases. If the reliability of network is increased through the end to end delivery, it will increase the cost. Therefore after considering all these factors, a new scheme is proposed in which video data is divided into different streams. Each stream moves from a different path [11].

\subsection{Quality of Service and Load Balancing}

In recent scenario the wireless mesh networks are widely used for transferring data from one end to other end. In these network gateways are present in which packets are going through gateways. Sometimes when the number of packets increases in the network and gateways are not able to cope up with the increase in the traffic as their buffer is saturated. This result in the dropping of packets which are not desirable because packets which are present in the network have already consumed lot of resources. A new approach is introduced in which all these limitations are removed. In this approach the load in the network is balanced by finding the gateways starting from source to destination due to this the quality of video is maintained in wireless mesh networks[12]. The evaluation of quality of MPEG video transmissions in the network is analyzed. This scheme is good for large networks having relatively large topologies, different kinds of traffic, nodes mobility. In this, the quality of the service is evaluated in the PSNR assessment metric with reasonable accuracy. This evaluation framework is responsible for the networking and video coding techniques for most of cases [27].

Table1. Comparison of various approaches

\begin{tabular}{|c|c|c|c|c|c|c|}
\hline APPROACHES & $\begin{array}{l}\text { NO. OF } \\
\text { NODES }\end{array}$ & $\begin{array}{l}\text { NO. OF } \\
\text { FRAMES }\end{array}$ & $\begin{array}{c}\text { PSNR } \\
(\text { db })\end{array}$ & THROUGHPUT & $\begin{array}{c}\text { MOS } \\
\text { (MEAN } \\
\text { OPINION } \\
\text { SCORE) }\end{array}$ & $\begin{array}{l}\text { SIMULATION } \\
\text { ENVIRONMENT }\end{array}$ \\
\hline $\begin{array}{l}\text { Application centric } \\
\text { approach [6] }\end{array}$ & - & 120 & 29 & - & - & Trellis graph \\
\hline DDR [8] & 25 & 150 & - & $98 \%$ & - & MATLAB \\
\hline ACE [14] & - & - & $>=37$ & - & 5 & NS2 \\
\hline OR [16] & 10 & - & 47 & $\begin{array}{l}0.4 \text { (normalized } \\
\text { effective } \\
\text { throughput) }\end{array}$ & - & Roofnet trace \\
\hline DCA [23] & 60 & - & - & $65 \mathrm{mbps}$ & - & GloMoSim \\
\hline DSDV [24] & 100 & - & - & $6.5 \mathrm{mbps}$ & - & NS2 \\
\hline PEMuR [26] & 300 & 300 & 39 & - & - & MATLAB/SIMULINK \\
\hline FV [27] & - & - & $>=37$ & - & 5 & EVAL ID AND NS2 \\
\hline $\begin{array}{c}\text { ADAPTIVE } \\
\text { REDUNDANCY } \\
{[29]}\end{array}$ & - & 100 & 39 & - & - & NS2 SIMULATOR \\
\hline DD VIDEO [30] & 100 & - & 29.809 & - & - & $\begin{array}{l}\text { C PROGRAM AND } \\
\text { LINDO API } 3.0\end{array}$ \\
\hline
\end{tabular}




\subsection{Scheduling}

Recently multiple descriptions are widely used to send the video data from source to destination but here the issue arises that due to presence of errors the quality of the video is affected. Also, the multiple description streams have competition among themselves for acquiring the limited resources in the wireless multi hop networks. So, by considering all these issues and problems, a new scheme is introduced in which the video data is send by multi path routing from source to destination. The multi path routing and multiple description rate control both works together to form the optimization of the network to reduce the competition among different multiple description streams. First source sends the messages for the path discovery and intermediate nodes have the data of congestion which is known as congestion price and when the destination receives these messages the source decides the best possible path to destination [13].

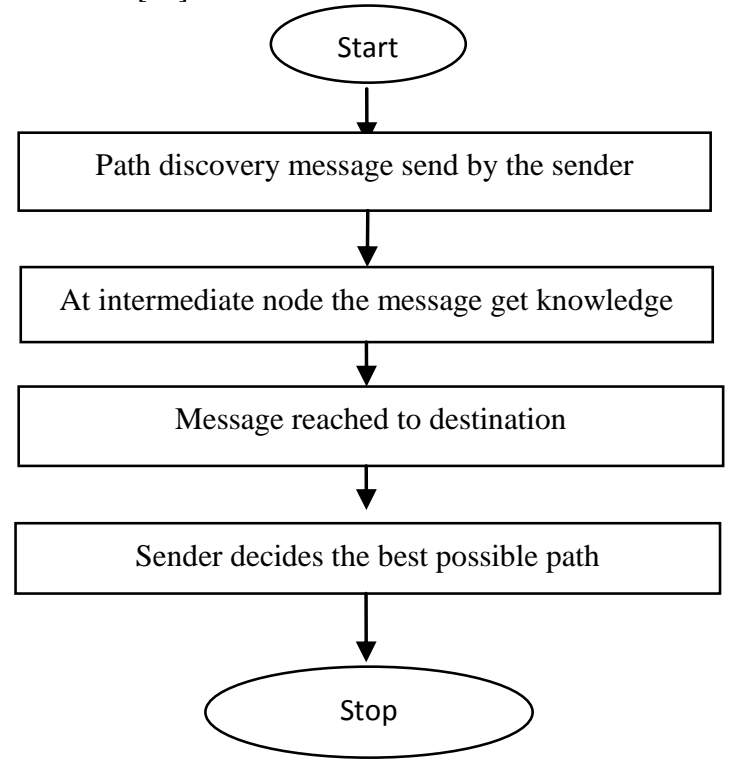

Fig 2: Path discovery method

\subsection{Model Based Schemes}

In recent scenario the security of video data is an important concern. There are various ways to provide the security to video data e.g. encryption. In this, the video data is encrypted so that the data is safe from intruders. There are lot of encryption schemes present for the encryption of video data but after encryption the size of video data increases which affect adversely during the transmission of video. Some of the traditional encryption algorithms like RSA and DES are present but complexity of the computation and larger size of data makes this algorithm inefficient for encrypting video data. Therefore a new encryption algorithm is introduced which is based on reducing the size of video data. This algorithm is applied on pay per view application [19].

\subsection{Energy Efficient}

The recent techniques improve the communication widely. The audio-video information is available at a very minimal cost. Wireless multimedia networks have different applications which are multimedia based. In video communication, quality of service is maximized and energy is minimized. The new scheme is proposed which is energy efficient and minimizes the energy expenditures in video communication and hence saves energy. This scheme is based on energy efficient hierarchical routing and video packet scheduling algorithm. This algorithm chooses best possible energy efficient path for the transfer of video packets and it manages the network in such a way that the useless data is not transmitted due to energy threshold. The advantage of scheduling algorithm is that it enables the reduction in video transmission rate by the minimum increase in distortion. It also predicts the resulted video distortion due to any error pattern [26].

\section{MAC SUPPORT}

\subsection{Routing Framework}

Today mobile ad hoc networks are used for communication .It is very challenging to improve the capacity of wireless networks. IEEE 802.1 standard allows seeing multiple channels at the physical layer. In mobile ad hoc routing, protocols are used to run on one channel. In this, new routing approach is developed in which the throughput increases by increasing the frequency and temporal resources to ad hoc networks. The new scheme also searches the best possible path in the environment where radio interfaces and available channels are limited. It explores the resources at the MAC layer for the efficient usage of the channel [23].

\subsection{DSDV}

In wireless mobile ad hoc networks IEEE 802.11 MAC protocol is used which is made for sharing of single channel. Due to single channel, the throughput of the channel degrades and load balancing is not possible. There are many IEEE 802.11 physical protocols present which allow the different multipath between source to destination therefore simultaneous messages has been sent to the receiver which increases the network throughput. Therefore a new routing protocol is designed in which multiple channels are present for simultaneous transmission and reception [24].

\section{COMPARISION OF VARIOUS APPROACHES}

Various approaches are compared in the table 1. The approaches are summarized for various parameters like number of nodes, number of frames, PSNR, throughput and mean opinion score. The ACE approach [14] has the maximum value of the PSNR which depicts the high quality of video and the presence of the noise is less. In case of DDR approach [8] maximum throughput is gained.

\section{CONCLUSION AND FUTURE WORK}

In this paper video transmission in the Internet scenario, where packets move from source to destination, has been studied. Various methods and schemes are discussed to send the packet from source to destination efficiently. Also, various methods like encryption, scrambling techniques are used to hide the content from the intruders. The algorithms are compared on various parameters like delay, PSNR, throughput to provide the smooth playback of video at the destination side. These algorithms are also efficient to send the video data securely from source to destination. The problem of video copy attack still exists where an intruder can copy all the coming packets and extract frames from them. If the sequence of packets is same as sequence of frames then whole video can be thus be copied. In future, algorithms preventing the packet copy attack on the video data in the network should be implemented so that data can transfer efficiently. 


\section{ACKNOWLEDGMENTS}

I am thankful to my guides for their guidance and support.

\section{REFERENCES}

[1] SeongOunHwang,"Content and Service Protection for IPTV," Broadcasting, IEEETransactions on, vol.55, no.2, pp.425,436, June 2009.

[2] Jeffrey, M.; Park, S.; Lee, K.; Adams, G.; Savage, S., "Content security for IPTV," Communications Magazine, IEEE , vol.46, no.11, pp.138,146, November 2008.

[3] Rajpurohit, J.; Khunteta, A.,"A scalable frame scrambling algorithm for video encryption," Information \&Communication Technologies (ICT), 2013 IEEE Conference on ,vol.,no., pp.981,985, 11-12 April 2013.

[4] Alattar, A.M.; Al-Regib, G.I., "Evaluation of selective encryption techniques for secure transmission of MPEG compressed bit-streams,"Circuits and Systems, 1999. ISCAS '99.Proceedings of the 1999 IEEE International Symposium on, vol.4, no., pp.340,343 vol.4,Jul 1999.

[5] Alattar, A.M.; Al-Regib, G.I.; Al-Semari, S.A.,'Improved selective encryption techniques for secure transmission of MPEG video bit-streams," Image Processing, 1999. ICIP99.Proceedings. 1999 International Conference on , vol.4, no., pp.256,260 vol.4, 1999.

[6] Dalei Wu; Song Ci; Haohong Wang; Katsaggelos, A.K., "Application-Centric Routingfor Video Streaming Over MultiHop Wireless Networks,"Circuits and Systems for VideoTechnology, IEEE Transactions on , vol.20, no.12, pp.1721,1734, Dec. 2010.

[7] Gharavi, H.,"Multi-channel for multihop communication links," Telecommunications, 2008.ICT 2008. International Conference on , vol., no., pp.1,6, 16-19 June 2008 .

[8] Gharavi, H.,"Multichannel Mobile Ad Hoc Links for Multimedia Communications, "Proceedings of the IEEE, vol.96, no.1, pp.77,96, Jan. 2008.

[9] Khalek, A.A.; Dawy, Z., "Optimized resource allocation for video transmission overand Computer Networks (SoftCOM), 2011 19th International Conference on , vol., no.,pp.1,6, 15-17 Sept. 2011.

[10] Bo Rong; Yi Qian; Kejie Lu; Hu, R.Q.; Kadoch, M., "Multipath routing over wirelessmesh networks for multiple description video transmission," Selected Areas in Communications,IEEE Journal on , vol.28, no.3, pp.321,331, April 2010.

[11] Qaisar, S.B.; Radha, H., "Multipath multi-stream distributed reliable video delivery in Wireless Sensor Networks," Information Sciences and Systems, 2009. CISS 2009. $43^{\text {rd }}$ Annual Conference on , vol., no., pp.207,212, 18-20 March 2009.

[12] Chungui Liu; YantaiShu; Lianfang Zhang; Jun Li, "Efficient Multiple Gateways Load-Balancing and QoS Routing in Wireless Mesh Networks," Wireless Communications, Networkingand Mobile Computing, 2008. WiCOM '08.4th International Conference on, vol., no., pp.1,4, 12-14 Oct. 2008.
[13] Liang Zhou; Xinbing Wang; Yajun Li; BaoyuZheng; Geller, B., "Optimal schedulingfor multiple description video streams in wireless multihop networks," CommunicationsLetters, IEEE , vol.13, no.7, pp.534,536, July 2009.

[14] YuweiXu; Deng, J.D.; Nowostawski, M., "Optimizing Routing in Multi-hop WirelessNetworks Using Analytical Capacity Estimation: A Study on Video Streaming," High PerformanceComputing and Communication \& 2012 IEEE 9th International Conference onEmbedded Software and Systems (HPCCICESS), 2012 IEEE 14th International Conferenceon , vol., no., pp.748,755, 25-27 June 2012.

[15] do Rosario, D.; Costa, R.; Paraense, H.; Machado, K.; Cerqueira, E.; Braun, T., "Asmart multi-hop hierarchical routing protocol for efficient video communication over wirelessmultimedia sensor networks,"Communications (ICC), 2012 IEEE International Conferenceon , vol., no., pp.6530,6534, 10-15 June 2012

[16] Mei-Hsuan Lu; Steenkiste, P.; Tsuhan Chen, "Video transmission over wireless multihop networks using opportunistic routing," Packet Video 2007, vol., no., pp.52,61, 12-13 Nov. 2007.

[17] Shi,Changgui, Sheng-Yih Wang, and Bharat Bhargava"MPEG video encryption in real-time using secret key cryptography." in Proc. Int. Conf. Parallel and DistributedProcessing Techniques and Applications. 1999.

[18] Changgui Shi; Bhargava, B., "An efficient MPEG video encryption algorithm," Reliable Distributed Systems, 1998. Proceedings. Seventeenth IEEE Symposium on, vol., no.,pp.381,386, 20-23 Oct 1998.

[19] Vino, T.; Logashanmugam, E., "A model-based multimedia Encryption scheme forreal time videos," Recent Advances in Space Technology Services and Climate Change(RSTSCC), 2010 , vol., no., pp.171,173, 13-15 Nov. 2010

[20] Shujun Li; Guanrong Chen; Cheung, A.; Bhargava, B.; Kwok-Tung Lo, "On theDesign of Perceptual MPEGVideo Encryption Algorithms," Circuits and Systems for VideoTechnology, IEEE Transactions on , vol.17, no.2, pp.214,223, Feb. 2007.

[21] Wang; Hong-Bin Yu; MengZheng, "A DCT-based MPEG-2 transparent scrambling algorithm,"Consumer Electronics, IEEE Transactions on , vol.49, no.4, pp.1208,1213, Nov.2003.

[22] Ramamurthi, V.; Vadrevu, S.K.C.;Chaudhry, A.; Bhatnagar, M.R.,"MulticastCapacityof Multi-Channel MultihopWireless Networks," Wireless Communications and NetworkingConference, 2009. WCNC 2009.IEEE, vol., no., pp.1,6, 5-8 April 2009.

[23] Jinhua Zhu; Xin Wang; DahaiXu, "A Unified MAC and Routing Framework forMultichannel Multi-interface Ad Hoc Networks," Vehicular Technology, IEEE Transactionson, vol.59, no.9, pp.4589,4601, Nov. 2010.

[24] UngheeLee; Midkiff, S.F.; Park, J.S.,"A proactive routing protocol for multi-channelwireless ad-hoc networks (DSDV-MC)," Information Technology: Coding and Computing,2005. ITCC 2005. International Conference on, vol.2, no., pp.710,715 Vol. 2, 4-6 April2005. 
[25] Swaminathan, V.; Mitra, S., "A partial encryption scheme for AVC video," EmergingSignal Processing Applications (ESPA), 2012 IEEE International Conference on , vol., no.,pp.1,4, 12-14 Jan. 2012.

[26] D. Kandris, M. Tsagkaropoulos, I. Politis, A. Tzes, and S. Kotsopoulos,Energyefficientand perceived qos aware video routing over wireless multimedia sensor networks, AdHoc Networks, 2011.

[27] K. Chih-Heng, S. CeKuen, H. Wen-Shyang, and A. Ziviani,An evaluation frameworkfor more realistic simulations of MPEG video transmission. Journal of Information Scienceand Engineering, vol. 24, no. 2, pp. 425 440, 2008.
[28] Zhenyong Chen, Zhang Xiong, and Long Tang, "A novel scrambling scheme for digitalvideo encryption", in Proc.of Pacific-Rim Symposium on Image and Video Technology(PSIVT), 2006, pp. 9971006.

[29] E. Akyol, A. M. ekalp, and M. R. Civanlar, A flexible multiple description codingframework for adaptive peer to-peer video streaming, IEEE J. Select. Topics Signal Processing,vol. 1, no. 2, pp. 231-245, 2007.

[30] S. Kompella, S. Mao, Y. T. Hou, and H. D. Sherali, Cross-layer optimized multipath routing for video communications in wireless networks, IEEE J. Select. Areas Commun.,vol. 25, no. 4, pp. 831-840, May 2007. 